um die Anwendung der Konvention auch auf Länder- und Kommunalebene überprüfen und zu gewährleisten und in der Folge eine entsprechende Berichterstattung an den Ausschuss sicherzustellen, die dem föderativen Charakter unserer Republik Genüge tut (Pkte. 12-16 der Abschließenden Beobachtungen des Ausschusses). Gute Beispiele geben hier die Staatenberichte von Kanada oder Australien. Welche Schritte wird die Bundesregierung hier unternehmen?

- Die vom CEDAW-Ausschuss kritisierte unzureichende Ausstattung und die unzureichenden Kompetenzen der Antidiskriminierungsstelle einschließlich der Frage ihrer Unabhängigkeit sind ebenfalls Punkte, die vom djb seit Inkrafttreten des AGG kritisch gesehen werden (Pkte. 17-20 und 35-36). Sollten die Erfahrungen mit dem AGG - insbesondere die tatsächlich ausgebliebene Klagewelle - nicht genutzt werden, um hier entsprechende Änderungen im Gesetz auf den Weg zu bringen?

- Ganz konkrete Forderungen kommen aus dem Ausschuss für den Einsatz zeitlich befristeter Sondermaßnahmen aufgrund des erschreckend geringen Anteils von Frauen in Führungspositionen sowohl im öffentlichen Leben (hier steht Deutschland an drittletzter Stelle in Europa), als auch in der Privatwirtschaft (Pkte. 13-14 und 25-26). Ausdrücklich genannt werden die auch vom djb schon mehrfach geforderte
Einführung von Quoten, von Anreizsystemen und der Erlass eines Gleichstellungsgesetzes für die Privatwirtschaft. Der deutliche internationale Druck stellt ein sehr gutes zusätzliches Argument dar, um hier endlich die notwendigen Schritte einzuleiten.

- Einen großen Raum nahm in den Ausschussverhandlungen die Arbeitsmarktsituation von Frauen und die damit zusammenhängende Lohnlücke zwischen Frauen und Männern ein (Pkte. 13-14 und 33-36). In engem Zusammenhang hiermit stehen die kritischen Anmerkungen des CEDAWAusschusses zur diskriminierenden Situation von Frauen im Sozialleistungs- und Rentenbezug (Pkte. 37-38). Zu diesem gesamten Themenkomplex gibt es auch angesichts des geforderten Zwischenberichts für den CEDAW-Ausschuss dringenden Handlungsbedarf.

- Auch zu den vom Ausschuss angesprochenen Fragen zur Situation von Gewalt betroffenen Frauen und der Finanzierung von Schutzmöglichkeiten dieser Frauen (Pkte. 41-46 und 59-60) hat der djb mehrfach Vorschläge geäußert. Wird es hierzu weitere Maßnahmen der Bundesregierung geben? Dringlich scheint auch die Umsetzung der auch in früheren Jahren geäußerten Empfehlungen des Ausschusses hinsichtlich aussagekräftiger Statistiken in bestimmten Aspekten dieses Bereichs (Pkte. 45-46).

\title{
Arbeitsmarkt und Zuwanderung
}

\section{Hohenheimer Tage zum Ausländerrecht 2009 \\ 30. Januar bis 1. Februar 2009 in Stuttgart-Hohenheim}

Die diesjährigen Hohenheimer Tage zum Ausländerrecht, die traditionell am letzten Januar-Wochenende in der Akademie der Diözese RottenburgStuttgart stattfinden, waren dem Thema „Arbeitsmarkt und Zuwanderung “ gewidmet. Wieder einmal hatten sich etwa 250 Teilnehmerinnen und Teilnehmer aus Justiz und Anwaltschaft, aus Politik und Kirche, aus Medien und Wissenschaft sowie aus Menschenrechtsorganisationen und Flüchtlingsverbänden zu einem Austausch eingefunden. Vor dem Hintergrund des am 1. Januar in Kraft getretenen Arbeitsmigrationsförderungsgesetzes ${ }^{1}$ standen Fragen der Arbeitsmigration und ihre Auswirkungen auf den Arbeitsmarkt und den Aufenthaltsstatus der Betroffenen im Zentrum der Veranstaltung. Daneben diente der Austausch auch wieder einer Standortbestimmung zu allen anderen Fragen des Ausländerrechts.

Mit ihrem Einführungsreferat stellte die Beauftragte der Bundesregierung für Migration, Flüchtlinge und Integration, Staatsministerin Prof. Dr. Maria Böhmer, die Ergebnisse des Nationalen Inte- grationsplanes vor und verwies auf die in den letzten Jahren erreichten Erfolge zum Beispiel im Bereich der Bleiberechtsregelungen, auf die Rechtsänderungen im Bereich des BAföG, die nun auch geduldeten Ausländern die Zugang zu Ausbildungsförderung ermöglichen, aber auch auf zukünftige soziale Herausforderungen wie die Regelung des Schulbesuchs für Illegale und die Arbeit in den Wohngebieten in Form eines „Quartiersmanagements“. Sie plädierte für die Fortsetzung des Integrationsplanes als strukturiertem Dialog zwischen allen Beteiligten und kündigte einen indikatorengestützten Prozessbericht zur Evaluation der Ziele des Integrationsplanes an. Sie betonte, dass der $\mathrm{Zu}$ gang zu Arbeit und Ausbildung ebenso wie die Anerkennung ausländischer Bildungs- und Berufsabschlüsse hierbei ein Schwerpunkt seien.

Prof. em. Dr. Kees Groenendijk, Nijmwegen, der den Hohenheimer Tagen als Referent insbesondere für die europarechtlichen Fragen der Migration

G. v. 20.12.2008 (BGBI. I 2008, S. 2846).
Susanne Walter Richterin am OVG, Hamburg

\section{Gabriela} Lünsmann Rechtsanwältin, Kanzlei „Menschen und Rechte", Hamburg Beide Mitglied der Kommission Öffentliches Recht, Europaund Völkerrecht des djb 
weiterhin verbunden ist, bewertete die von ihm ausgemachten „Dilemmata“ der europäischen Einwanderungspolitik. In einer rechtsvergleichenden Analyse stellte er das Arbeitsmigrationssteuerungsgesetz in einen Zusammenhang mit den bestehenden Richtlinien der EU, die einen Zugang zum Arbeitsmarkt ausdrücklich bisher nicht regeln. Die Interessen der Mitgliedstaaten seien gespalten: Sie wollten zum einen eine restriktive Einwanderungspolitik, sie wollten Arbeitsmigranten, aber keine Arbeitsmigration, sie wünschen eine gemeinsame Arbeitsmigrationspolitik, wollten aber ihre Eigenständigkeit behalten.

Der Zugang zu Arbeit ist für Drittstaatsangehörige bisher in anderen Richtlinien geregelt, die „Blue Card“ stellt lediglich eine Privilegierung für Hochqualifizierte auch hinsichtlich des Familiennachzugs dar. Auf der politischen Ebene hatte Frankreichs Ministerpräsident Sarkozy im Oktober 2008 einen Nationalen Pakt zur Einwanderung angestoßen. Zu geplanten Maßnahmen hat der Ministerrat Beschlüsse gefasst, denen das Europäische Parlament bereits zugestimmt hat. Danach soll generell der Zugang in die Mitgliedstaaten begrenzt werden. Daneben gelten die nationalen Voraussetzungen als alternative Rechtsgrundlagen für den Aufenthalt weiter. Illegale Beschäftigung soll weiter sanktioniert werden durch eine Arbeitsplatzprüfung. Die Gewerkschaften können Nachzahlungsverfahren betreiben, wenn die Voraussetzungen ordnungsgemäßer Entlohnung und Arbeitsbedingungen nicht vorliegen. Gleichzeitig droht der oder dem Betroffenen die Ausweisung. Schwerpunkt der Politik bleibt weiter die zirkuläre Migration.

Als Fazit formulierte der Referent, dass die Zulassung von Einwanderung zum Zweck der Arbeitsaufnahme weiter in der Kompetenz der Mitgliedstaaten bleiben und dass Mobilität allein über die Richtlinie 2003/109/EG² oder über die Einbürgerung erfolgen solle.

Der sich anschließende Vortrag von Prof. Dr. Herbert Brücker, IAB Nürnberg/Universität Bamberg, war den volkswirtschaftlichen Fragen der Arbeitsmigration gewidmet. Brücker stellte zunächst die (zwangsweisen) Anreize zur Migration dar, wie sie bereits durch den Fall des „Eisernen Vorhangs“, durch die Bürgerkriege in Ex-Jugoslawien in den 90er Jahren und die EU-Osterweiterung erfolgt sind. Wegen des Anwerbestopps existierte zuvor in der Bundesrepublik seit 1973 ein faktischer Arbeitsmarktschutz, der nur durch Familiennachzug und humanitäre Migration „durchbrochen“ wurde.

18 Prozent der Beschäftigten in der Bundesrepublik haben heute einen Migrationshintergrund. Die Anreize zur Arbeitsmigration nehmen wegen der demografischen Besonderheiten ständig zu, das Potential der Erwerbspersonen wird bis 2050 stetig sinken. Auch der - im AufenthG nicht umgesetzte - Vorschlag der Süssmuth-Kommission, eine gezielte Arbeitsmigration (im Umfang von etwa 200.000 Personen) zuzulassen, hätte an der Gesamtqualifikation und am Gesamtbedarf der Arbeitskräfte in der Zukunft wenig geändert.

Besonders bemerkenswert war die These des Referenten, gegenwärtig sei das Bildungsniveau der Arbeitsmigranten ge- ringer als das des Durchschnitts in der OECD und auch geringer als das ihrer Herkunftsländer. Prof. Dr. Brücker stellte dann das Ergebnis einer Studie seines Instituts vor, die den gesellschaftlichen Befürchtungen „Durch Lohnkonkurrenz steigt die Arbeitslosigkeit“ und „Ausweitung der Arbeitsmigration (um $1 \%$ ) führt zu niedrigeren Löhnen“ auf den Grund gehen sollte. Sie ergab, dass die Erhöhung der Arbeitsmigration allenfalls zu einer Flexibilität in einzelnen Bereichen des Arbeitsmarktes, nämlich bei jungen Arbeitnehmern und bei Hochund Geringqualifizierten, führen würde. Die Gesamteffekte beschrieb Brücker als gering, da Inländer und Ausländer selbst bei gleicher Ausbildung und Berufserfahrung nicht miteinander konkurrierten. Der Kapitalstock passe sich an die Nachfrage an, daher blieben Kapital und Output langfristig konstant. Die Migration sei insoweit neutral. Eine signifikante Auswirkung durch Zulassung von Migranten auf die Arbeitslosenquote gebe es nicht. Verlieren würden allenfalls Inländer ohne Berufsabschluss; weiterhin gebe es Effekte unter den Ausländern im Sinne einer „Ghettobildung“. Insgesamt stiegen die unternehmerischen Gewinne mit der Qualifikation der Zuwanderer.

Das Einführungsreferat des nächsten Tages war den neuen nationalen Regelungen gewidmet. Sybille Roeseler und Dr. Michael Maier-Borst stellten die Bestimmungen des Arbeitsmigrationssteuerungsgesetzes vor. Danach bleibt es bei einem Anwerbestopp nach $\mathbb{S} \mathbb{S} 19,21$ AufenthG bezogen auf den Außenzugang zum Arbeitsmarkt. Die Vorrangprüfung wird in den Fällen des $\mathbb{S} 18$ a Abs. 2 AufenthG und den $\mathbb{S} \mathbb{S} 6,9,10$ BeschVerfV abgeschafft. Der Arbeitsmarkt wird für geduldete Ausländer nach vier Jahren geöffnet; dies begünstigt Ausländer, die sich aufgrund des IMK-Bleiberechtsbeschlusses und der Regelung der $\$ \mathbb{S} 104 \mathrm{a}$ und 104b AufenthG bereits längere Zeit im Bundesgebiet aufhalten und/oder bereits einen Schulabschluss und/oder sonstige berufliche Qualifikationen erworben haben. Die Zugangsbeschränkungen für die neuen EUBeitrittsländer bestehen zunächst weiter. Die Änderung der BeschVerfV $(\mathbb{S} \mathbb{S} 3 \mathrm{a}, 10)$ ermöglicht den Verzicht auf die Vorrangprüfung bei der Beschäftigung von ausländischen Schülerinnen und Schülern, die im Inland einen Schulabschluss erworben oder an einer schulvorbereitenden Bildungsmaßnahme teilgenommen haben. Allerdings bedarf es weiterhin einer Prüfung der Gleichwertigkeit der Arbeitsbedingungen bei einer bereits erworbenen Berufsausbildung, z.B. um Lohndumping zu verhindern. $\mathbb{S} 18 \mathrm{a}$ AufenthG ermöglicht zudem einen Arbeitsmarktzugang für Geduldete, die eine Ausbildung oder ein Hochschulstudium abgeschlossen haben. Eine Verfestigung des Aufenthalts ist nach zwei Jahren möglich ( $\mathbb{S} 18 \mathrm{a}$ Abs. 2 AufenthG). Ein Anspruch auf Ausbildungsförderung nach dem BAföG besteht für seit mindestens vier Jahren geduldete Ausländer, wenn sie die Voraussetzungen des $\mathbb{} 8$ Abs. $2 \mathrm{a}$ BAföG erfüllen. Außerdem ist eine Förderung der Berufsausbildung nach $\$ 63$ SGB III möglich.

2 Richtlinie 2003/109/EG des Rates vom 25.11.2003 betreffend die Rechtsstellung der langfristig aufenthaltsberechtigten Drittstaatsangehörigen. 
Durch die Absenkung des Durchschnittsverdienstes nach $\mathbb{} 19$ Abs. 2 AufenthG auf ca. 63.000 Euro werden wenig Effekte für die Zuwanderung Hochqualifizierter erwartet, da das Durchschnittseinkommen in der Bundesrepublik bei ca. 28.000 Euro liegt. Eine „Gegensteuerung“ erfolgt durch die

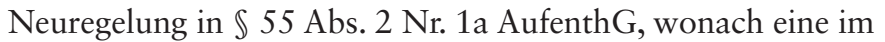
Ermessen stehende Ausweisung möglich ist, wenn die Ausländerin oder der Ausländer gegenüber dem Arbeitgeber falsche Angaben über ihren oder seinen Arbeitsvertrag gemacht hat. Problematisch ist in $\mathbb{S} 18 \mathrm{a}$ Nr. 1b) und c) AufenthG, dass es der Anerkennung eines ausländischen Bildungsabschlusses und damit der Prüfung der „Gleichwertigkeit“ bedarf. Auch stellt sich die Frage, ob der Bezug von BAföG als „öffentliche Mittel“ i.S.d. $\int 18$ a Abs. 1 Nr. 1c) anzusehen ist und damit entgegen der Intention der Regelung der Erteilung einer Aufenthaltserlaubnis entgegenstünde. Dieses Harmonisierungsproblem bei der Anwendung der verschiedenen Regelungen ist aber von Bund und Ländern bereits gesehen worden und wird vom Gesetzgeber korrigiert werden.

Jutta Driesch, Regionaldirektion der Bundesagentur für Arbeit, wies in ihrem anschließenden Vortrag auf die großen Erfolge hin, die in Baden-Württemberg für die Integration von Migranten - insbesondere jungen Migrantinnen und Migranten - am Arbeitsmarkt erreicht worden seien. In Baden-Württemberg habe jede/r dritte Erwachsene und jede/r vierte Jugendliche einen Migrationshintergrund. Die meisten Migrantinnen und Migranten kämen aus der Türkei und Italien. 41 Prozent hätten keinen Berufsabschluss, während die Qualifikationsanforderungen am Arbeitsmarkt weiter zunähmen. Driesch machte deshalb die Handlungsfelder der Bundesagentur aus: Netzwerkarbeit, Prävention und Stärkung der Berufskompetenz. Jedem Jugendlichen konnte im letzten Jahr ein Ausbildungsangebot gemacht werden. Die Referentin betonte, es sei dabei wichtig, die Eltern der Jugendlichen „mit ins Boot zu holen “, ebenso die Wirtschaft. Dafür würden im Jahr 2009 zehn Millionen Euro investiert. Auch müsse durch Sprachkurse die Qualifikation der Geringqualifizierten erhöht werden.

Die Wissenschaftlerin Dr. Bettina Englmann stellte im anschließenden Vortrag eine Studie über die Anerkennung ausländischer Bildungsabschlüsse in Deutschland vor dem Hintergrund der Lissabonner Anerkennungskonvention ${ }^{3}$ vor. Ausgenommen hiervon waren reglementierte Berufe wie Heilberufe. Für die Anerkennung wurden Ausbildungszeiten und -abschlüsse verglichen. Bei einer reinen Zeugnisbewertung fänden häufig nach der Schulzeit erworbene Kenntnisse und Kompetenzen keine Berücksichtigung. Auch gebe es Unterschiede in den Prüfungsanforderungen der einzelnen Länder zwischen Drittstaatsangehörigen und denjenigen, die einen Aussiedlerstatus hätten. Bei einer Teilanerkennung nach der EU-Anerkennungs-RL könne später ein Ausgleich durch eine Eignungsprüfung oder Lehrgänge erfolgen und so eine Vollanerkennung geschaffen werden. Bisher gebe es allerdings kaum klärende Gerichtsverfahren trotz Anerkennungsanträgen im vierstelligen Bereich. Gabriele Erpenbeck, Niedersächsisches Ministerium für Inneres, Sport und Integration, und Allan
Bruun Pedersen, CIRIUS, The Danish Ministry of Science, Technology and Innovation, sowie Gabriela Schierenbeck, Handwerkskammer Bremen, stellten jeweils für ihren Bereich die Zusammensetzung und besonderen Bedarfe der Arbeitssuchenden bzw. die Ambitionen der verschiedenen Träger bei der Anerkennung von Bildungsabschlüssen - zum Teil, wie in Bremen, auf weniger gesetzlich geregeltem als auf pragmatischem Wege - vor. Überzeugend war der dänische Weg einer zentralen staatlichen Agentur für die Anerkennung von Bildungsabschlüssen (CIRIUS), die aus der Diskussion um den „brain waste“ geboren wurde und verhindern soll, dass Migrantinnen und Migranten mit guter, im Heimatland erworbener Ausbildung Taxi fahren oder in andere Länder abwandern. Die Entscheidungen von CIRIUS basieren auf der Lissabonner Anerkennungskonvention und sind bindend. Die Anerkennung wird nur versagt, wenn es einen „substanziellen“ Unterschied zwischen dem im Drittland erlangten Bildungsabschluss und dem dänischen gibt. Dieser Ansatz, der von der Behörde den Beweis dieses Tatbestandes verlangt, kommt einer Beweislastumkehr gleich und beruht auf der Akzeptanz der Bewertungsautonomie des Heimatlandes. Verlangt wird nicht die vollständige Vergleichbarkeit mit dem dänischen Abschluss. Die Entscheidungen der Behörde beruhen auf gesammelten und bewerteten Kenntnissen der meisten in den zahlreichen Herkunftsländern erwerbbaren Bildungsabschlüsse. Sie sind im Internet abrufbar und ermöglichen für Arbeitgeber schnelle und einheitliche Informationen über den (Aus-)Bildungsstand potentieller Arbeitnehmer. Zugleich wies Pedersen aber darauf hin, dass mit der Anerkennung eines ausländischen Abschlusses nicht zugleich ein Job verbunden sei, da der Arbeitsmarkt nach dem „Tick-Trick-Track-Prinzip“ funktioniere: Referenzen und soziale Beziehungen oder Empfehlungen seien für den Erhalt eines Arbeitsplatzes bedeutender als die Anerkennung des mitgebrachten Abschlusses.

Wolfgang Kreissl-Dörfler, Mitglied des EP (Sozialisten) stellte im Anschluss die Vorstellungen und Einwirkungsmöglichkeiten des Europäischen Parlaments im Migrationsbereich dar und verwies auf den Vorstoß von Sarkozy und die Situation durch neue Außengrenzen in Europa, die die Interessen der neuen Mitgliedstaaten maßgeblich bestimme.

An diese Vorträge schlossen sich wie gewohnt in zwei Blöcken die verschiedenen „Foren“ an, in denen die Teilnehmerinnen und Teilnehmer der Tagung in Workshops Themen wie Arbeitsmigration von Drittstaatsangehörigen, die Deutsche Islamkonferenz, den Stand der neuen Rechtsprechung zum Ausländer- und Flüchtlingsrecht oder den Zugang zu Sozialleistungen für neue Unionsbürger vertiefen konnten. Danach konnten alle bei anregendem, aber auch entspannendem Jazz der schon seit einigen Jahren regelmäßig in Hohenheim gastierenden, hochkarätig besetzten „Hohenheim All-Stars“ den Tag ausklingen lassen.

\footnotetext{
3 Richtlinie 2005/36/EG des Europ. Parlaments und des Rates vom 7.9.2005 über die Anerkennung von Berufsqualifikationen; Gesetz zur Umsetzung der RL v. 2.12.2007 (BGBI I, S. 2686).
} 
Der Sonntag war der Thematik des Staatsangehörigkeitsrechts gewidmet. Prof. Dr. Gerard-Rene de Groot, Universität Maastricht, führte mit einem humorvoll akzentuierten Referat zum Staatsangehörigkeitserwerb durch das „ius soli“, wie er seit dem StAG 2000 auch für Kinder ausländischer Eltern mit entsprechendem Aufenthaltsstatus in der Bundesrepublik möglich ist, in die Thematik ein. Dabei stellte der Referent rechtsvergleichend die Regelungen der 15 alten EU-Länder, Österreichs und der Schweiz dar. Einige Länder haben ein „doppeltes ius soli“ geregelt: ist bereit ein ausländischer Elternteil z.B. in Belgien, Portugal oder Frankreich geboren, erwirbt das Kind, das in diesem Land geboren wird, die Staatsangehörigkeit des Landes. Nur Deutschland hat einen Optionszwang in \29 Abs. 1 StAG geregelt. 13 von 18 Staaten akzeptieren eine mehrfache Staatsangehörigkeit, in einzelnen Ländern kann die Aufgabe der Staatsangehörigkeit schlicht erklärt werden.

Das sich anschließende provokante Thema des Podiumsgesprächs „Abschaffung des Optionszwangs“ verlangte von den Teilnehmerinnen und Teilnehmern eine Positionierung zur gegenwärtigen Rechtslage, die für die ersten jungen Erwachsenen bald Realität wird. Der Richter am BVerwG Prof. Dr. Uwe Berlit hielt den Optionszwang zwar nicht für wünschenswert, wenn nicht gar für unsinnig, aber für verfassungsrechtlich zulässig. Prof. Dr. Kay Hailbronner wies darauf hin, dass in der Praxis das Ziel der gesetzlichen Regelung - eine Vermeidung der doppelten Staatsangehörigkeit - schwer zu erreichen sei. Der Vorsitzende der türkischen Gemeinde in Deutschland Kenan Kolat nannte die Regelung und die Tatsache, dass nun insbesondere türkische junge Erwachsene optieren müssten, für einen „integrationspolitischen Scherbenhaufen“. Diesen sei nicht zu vermitteln, warum einige ihrer Freunde ihre (durch Einbürgerung erworbene) deutsche Staatsangehörigkeit ohne weiteres behielten, sie aber im Fall „vergessener (und damit nicht ausgeübter) Option “ ihre deutsche Staatsangehörigkeit verlören. Auf diese Weise schaffe man nicht nur praktische Probleme wie fehlende türkische Meldepapiere, sondern auch zwei Identitäten. Dabei müsse anerkannt werden, dass die Person auf Grund ihrer Sozialisierung in Deutschland und ihrer ausländischen Wurzeln beide Identitäten in sich vereinige. Auf dieses Phänomen verwies auch Gabriele Erpenbeck (Zentralkomitee der Deutschen Katholiken). Sybille Laurischk (MdB, FDP) verlangte, dass ein neuer Diskurs zum Staatsangehörigkeitsrecht mit dem Ziel einer möglichen Gesetzesänderung angestoßen werde.

Mit diesem übereinstimmenden Appell an den Gesetzgeber schloss eine anregende Tagung in Stuttgart-Hohenheim, die wie immer von Klaus Barwig, Dr. Gisbert Brinkmann und Dr. Christoph Schumacher durch die Gewinnung interessanter Referentinnen und Referenten sehr gut vorbereitet und souverän geleitet wurde.

\section{GF2D/CRIFF - ein Frauenrechtsverband aus Togo}

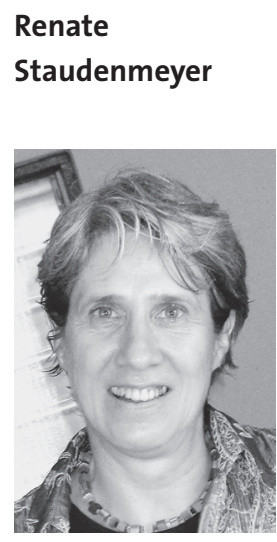

Fachkraft des DED (Deutscher Entwicklungsdienst), Lomé/Togo
Sylvia Cleff

Le Divellec, LL.M.

(Paris 2)

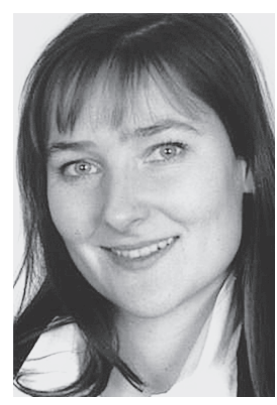

Mitglied der Kommission Öffentliches Recht, Europa- und Völkerrecht des djb, Beraterin/Juristin für die französische Unternehmensberatung „ALETEYA - conseil en diversité" in Paris
GF2D wurde 1992 als Nichtregierungsorganisation in Togo (Westafrika) von ca. 30 Juristinnen und Frauen mit unterschiedlichem beruflichem Hintergrund gegründet.

Nach der Erarbeitung einer Satzung hat sich dieser Frauenrechtsverband seit 1994 ein Exekutiv-Organ zur Seite gestellt, das „Centre de Recherche d'Information et de Formation pour la Femme“ (CRIFF). CRIFF ist damit beauftragt, diejenigen Aktivitäten umzusetzen, die in einem Fünfjahresplan für die drei Programmbereiche von GF2D konzipiert und geplant werden: für den Bereich juristische Dienstleistungen, den Bereich bürgerrechtliche Bildung von Frauen und den sozioökonomischen Bereich.

Der juristische Programmbereich von GF2D/CRIFF verfolgt die Verwirklichung übergeordneter Zielsetzungen, wie

- den Zugang von Frauen zum Recht, die Kenntnis ihrer (bürger-)rechtlichen Situation sowie deren effektive Nutzung zu maximieren,

- Frauenrechte auf nationaler Ebene allgemein verständlich zu vermitteln,

- die LaienjuristInnen so zu organisieren, dass die Wirkungen ihrer Aktivitäten durch die Verbesserung ihrer Arbeitsbedingungen optimiert werden,

- den rechtlichen Status von Frauen in Togo zu verbessern,

- geschlechtsspezifische Gewalt zu reduzieren, 